\title{
A LOW-COST PANORAMIC CAMERA FOR THE 3D DOCUMENTATION OF CONTAMINATED CRIME SCENES
}

\author{
D. Abate ${ }^{1,2}$, I. Toschi $^{3}$, C. Sturdy-Colls ${ }^{1}$, F. Remondino ${ }^{3}$ \\ ${ }^{1}$ Centre of Archaeology, Staffordshire University, Stoke on Trent, United Kingdom, (dante.abate, c.sturdy-colls)@staffs.ac.uk \\ ${ }^{2}$ Science and Technology in Archaeology Research Centre, STARC, The Cyprus Institute, Nicosia, Cyprus, d.abate@cyi.ac.cy \\ ${ }^{3}$ 3D Optical Metrology (3DOM) unit, Bruno Kessler Foundation (FBK), Trento, Italy, (toschi, remondino)@fbk.eu
}

\section{Commission II}

KEY WORDS: 360 cameras, Crime Scene Documentation, Photogrammetry, Feature Extractors

\begin{abstract}
:
Crime scene documentation is a fundamental task which has to be undertaken in a fast, accurate and reliable way, highlighting evidence which can be further used for ensuring justice for victims and for guaranteeing the successful prosecution of perpetrators. The main focus of this paper is on the documentation of a typical crime scene and on the rapid recording of any possible contamination that could have influenced its original appearance. A 3D reconstruction of the environment is first generated by processing panoramas acquired with the low-cost Ricoh Theta 360 camera, and further analysed to highlight potentials and limits of this emerging and consumer-grade technology. Then, a methodology is proposed for the rapid recording of changes occurring between the original and the contaminated crime scene. The approach is based on an automatic 3D feature-based data registration, followed by a cloud-to-cloud distance computation, given as input the 3D point clouds generated before and after e.g. the misplacement of evidence. All the algorithms adopted for panoramas pre-processing, photogrammetric 3D reconstruction, 3D geometry registration and analysis, are presented and currently available in open-source or low-cost software solutions.
\end{abstract}

\section{INTRODUCTION}

Before the advent of consumer-grade low-cost spherical panoramic sensors (e.g. Ricoh Tetha, Samsung Gear 360, LG360 cam, Nikon Key Mission 360, see Figure 1), panoramic photography and 3D modelling involved (i) stitching shots taken at different viewpoints into one single frame (Fangi, 2007; Fangi, 2009) or (ii) the use of very expensive rotating linear array cameras (Schneider and Maas, 2003; Amiri Parian and Gruen, 2010). The former approach needs precise acquisition and stitching procedures, that are often computationally and time consuming and do not always guarantee the final quality of the photogrammetric restitution due to various deficiencies which characterize the whole process (Szeliski and Shum, 1997; Sakharkar and Gupta, 2013). In contrast, today, available 360degree cameras can be used to create panoramic images or videos in real time, without the need of stitching or further processing and still providing high quality results. At the same time, with the development and increasing penetration of smartphone technologies, the camera processors and sensors have developed so rapidly that compact 360 -degree panoramic cameras have now become more affordable.

From the processing side, spherical cameras are today available among the camera calibration models implemented in a few low-cost (e.g. PhotoScan by Agisoft, Pix4Dmapper by Pix4D) and open source (e.g. COLMAP, mapillary) software solutions. This has further triggered the exploitation of 360-degree cameras for $3 \mathrm{D}$ reconstruction applications by the scientific community, as shown by the increasing number of papers aimed at testing the performance of these cheap image-based solutions (Aghayari et al, 2017; Barazzetti et al., 2017; Matzen et al. 2017; Perfetti et al., 2017).

In this study, the performances of the Ricoh Theta SC series camera (Figure 1a) for image-based rapid documentation and $3 \mathrm{D}$ reconstruction are tested within the forensic scenario, where a quick and exhaustive crime scene $3 \mathrm{D}$ recording is fundamental to avoid missing evidence. Indeed, the application of such low-cost and easily deployable technology is timely and primary in the current research context. Besides this, and supporting the sensor component, a great benefit to this sector is given by the present level of maturity and reliability of 3D image-based modelling techniques. In particular, their application shows great potentials in fields such as forensic investigation, where the rapid (and, potentially, photo-textured) $3 \mathrm{D}$ reconstruction of evidence is crucial for the decision-making processes.

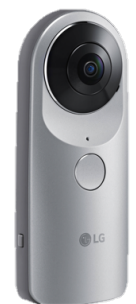

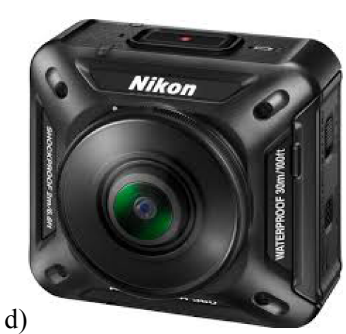

Figure 1. Examples of low-cost panoramic cameras available on the market. From left to right: Ricoh Theta, Samsung Gear 360, LG360 cam, Nikon Key Mission 360. 


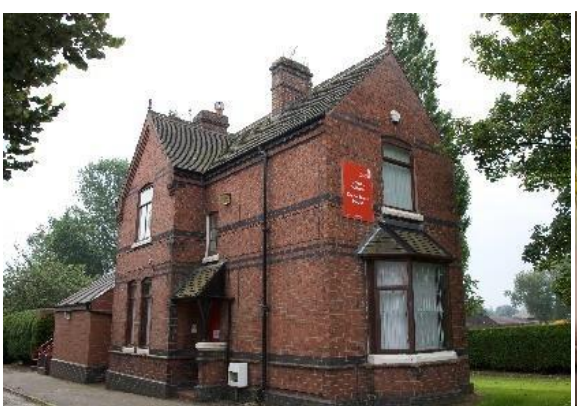

(a)

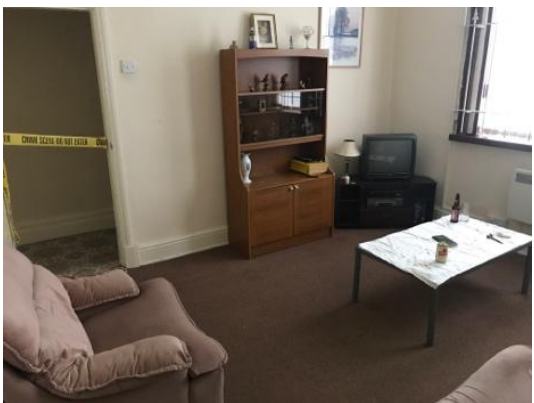

(b)

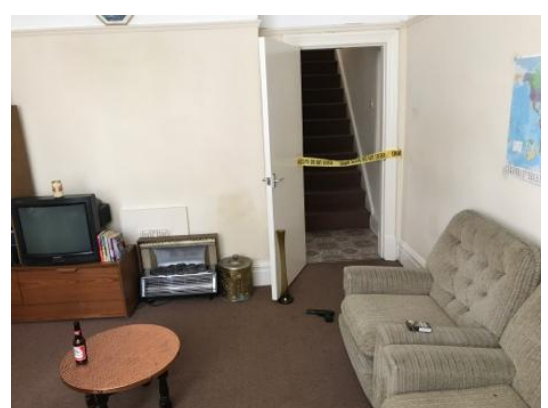

(c)

Figure 2. The Crime Scene House at the Staffordshire University (UK):

an overview of the building (a) and two views of the interior rooms (b and c).

\subsection{The Ricoh Theta SC 360 Camera}

At present, there are two distinct types of consumer-friendly 360 -degree cameras available on the market. One is equipped with single-lens whereas the other features a dual-lens configuration. Among the latter, the cheap and compact spherical panoramic camera Ricoh Theta (SC model) is rapidly gaining in popularity. It consists of two lenses (seven elements in six groups) which cover the entire scene by $360 \times 180$ degrees. The camera is equipped with a 12 Megapixel CMOS optical sensor, with $6.17 \times 4.55 \mathrm{~mm}$ size. It features a fixed aperture (f/2.0) and fixed focal length $(1.3 \mathrm{~mm}, 7.3 \mathrm{~mm} 35 \mathrm{~mm}-$ equivalent) lens.

The single images are stitched together using embedded software and there is no possibility to access the raw prestitching shots in the current version of camera. Indeed, images are currently exported in equi-rectangular JPEG format $(5376 \mathrm{x}$ 2688 pixels). The main technical specifications of the camera are listed in Table 1.

\begin{tabular}{|c|c|}
\hline Dimensions (mm) & $45(\mathrm{~W}) \times 131(\mathrm{H}) \times 23(\mathrm{D})$ \\
\hline Weight (g) & 102 \\
\hline Sensor size (mm) & $6.17 \times 4.55$ \\
\hline Image size (pixel) & $5376 \times 2688$ \\
\hline Focal length (mm) & $1.3(35 \mathrm{~mm}$-equivalent: 7.3$)$ \\
\hline Aperture & $\mathrm{f} / 2.0$ (fixed) \\
\hline Depth of field & $10 \mathrm{~cm}$ to infinity \\
\hline ISO sensitivity & $100-1600$ \\
\hline
\end{tabular}

Table 1. Main technical specifications of Ricoh Theta SC (source: www.theta360.com).

\subsection{The Crime Scene House}

The Crime Scene House is a structure built at the Staffordshire University (UK), to simulate a typical domestic home for crime scene investigation exercises. It is a detached house (Figure 2a) with a large garden and off-road parking. It contains seven rooms (examples are shown in Figure $2 b$ and $2 c$ ) that have been furnished to resemble a typical domestic home. There are also some permanent exhibits of crime scene investigation, e.g. examples of various types of bloodstain patterns. The extensive outside areas enable to simulate outdoor, such as clandestine burials, and vehicle crime scenes.

Conceived as a training and learning facility for students and researcher, the main function of the Crime Scene House is to simulate investigations in a realistic setting. Various types of scenarios can be set up and processed, using the appropriate equipment and procedures to prevent contamination and loss, and to ensure continuity of evidences.

\subsection{Paper objectives and structure}

The paper investigates the use of the Ricoh Theta spherical camera for the 3D documentation and the rapid identification of possible contaminations of a crime scene. The aim of the research is twofold: (i) to evaluate the performances of a lowcost imaging sensor for a quick $3 \mathrm{D}$ reconstruction of a forensic scenario and (ii) to propose a methodology for the rapid recording of possible contaminations which could have changed the original appearance of the crime scene. Indeed, a "missing evidence" scenario is simulated, by intentionally removing different objects originally present in the scene.

After a review of recent works dealing with forensic documentation in Section 2, the project is introduced in Section 3 , where the case study and the acquired data are described. An overview of the methodology applied for 3D geometry reconstruction and automatic registration of pre- and postcontamination data is given in Section 4, whereas results are analysed in Section 5. Finally, a discussion and some concluding remarks are drawn in Section 6.

\section{CRIME SCENE DOCUMENTATION}

\section{$2.1 \quad$ Related works}

Traditionally, police officers, crime scene technicians and researchers (in the case of historic crime) have focused on documenting a crime scene, removing and securing evidence. However, during this process, valuable proof may be lost or accidentally destroyed. Under the pressure of the time limitations imposed in some forensic investigations, scene examiners may also fail to adequately identify and document all of the elements that are present (Jackson and Jackson, 2008; Dutelle, 2016). Indeed, the position and location of evidence is crucial to an investigation and it is essential that such information is accurately recorded and documented.

The latest approaches of documenting and presenting crime scenes and associated material can today exploit a wide range of active and passive sensors and non-contact 3D imaging methods in order to address these challenges. 3D (and 4D) image-based and range-based modelling techniques provide indeed a fast, reliable and accurate way to document and acquire reality-based data without hampering investigations.

\subsubsection{Active and passive 3D imaging systems}

Active sensors, such as Terrestrial Laser Scanners (TLS), have proven to be successful in a wide range of applications, such as heritage documentation, reverse engineering, land mapping, and, lately, forensic science. Since traditional forensic documentation methods can be costly and time consuming without ensuring a detailed scene digitization, in the last decade 3D technology has provided a valid and valuable resource. 
Indeed, the use of laser scanner technology for the documentation of crime and collision scenes has significantly grown, as shown by the extensive body of literature in this field (Buck et al., 2014; Liscio et al., 2016; Dustin et al., 2016). For instance, in Holowko et al. (2016) a multi-resolution 3D recording technique is illustrated for the documentation of bloodstain pattern using a hierarchical 3D scanning platform, designed by following a top-down approach inspired by the traditional forensic photography.

Among the image-based approaches, a comparative study exploiting a 360-camera is proposed in (Sheppard et al., 2017). The authors investigate the accuracy of the adopted system in a mock crime scene and assessed the results in comparison with forensic documentation standards. The image-based method proposed by Zancajo-Blanquez et al. (2015) is instead applied to the forensic infography technique, which aims to relate all the signs in order to determine and demonstrate the facts related to a crime scenario. Another novel application can be found in the work of Thali, M.J. (2000), that successfully applied photogrammetric techniques for the documentation of forensicrelevant injuries. The study has shown how it is possible to reconstruct and match tyre tracks marks on body parts after an accident occurs, through image-based modelling techniques combined with CAD-based tools.

\section{PROJECT DESCRIPTION}

\subsection{Simulated crime scene scenarios}

The proposed methodology for the documentation of contaminated crime scenes is tested by setting up a mock environment at the Crime Scene House. Among its rooms, the "studio" is chosen for the experiments (Figure 2c). The scene features dimensions of approx. $4 \times 4 \times 3 \mathrm{~m}$ and is characterized by traditional furniture and decorations. Besides the presence of some paintings, the walls and the ceiling mostly have textureless and bright surfaces. Furthermore, un-controlled and un-diffused illumination conditions are provided by a single big window. Several small objects are intentionally scattered into the environment, i.e.:

- a gun replica,

- a knife replica,

- three drug replicas,

- a bottle,

- two cans.

Two different scenarios are finally simulated, as follows:

- Scenario A: all objects are randomly placed in the room on different positions and height levels;

- Scenario B: some of these objects (i.e. the gun, one can and a pills container) are removed from the scene.

\subsection{Image acquisition}

During the image-acquisition phase, the Ricoh Theta panoramic camera is fixed on a tripod and remotely operated through the dedicated smartphone app. Due to the device configuration, the only adjustable camera parameters are ISO sensitivity and shutter speed, which are set to 100 and $1 / 15 \mathrm{~ms}$, respectively.

Since the room features several distributed pieces of furniture (e.g. tables, armchairs and bookcases) and the objects are randomly placed on them and on the floor, many partiallyoccluded areas should be dealt with. In order to cope with this issue and ensure a good scene coverage and network stability, a camera network of twenty panoramas is carefully planned in advance, resulting in a mean camera-to-camera distance of about $1 \mathrm{~m}$. Although the same number of panoramas is recorded in both crime scene scenarios, the camera network is slightly changed among them in order to simulate real documentation conditions. Indeed, after the scene is first recorded when the crime event is disclosed (corresponding to Scenario A), the following scenario (B) will be potentially acquired when a reasonable doubt of contamination exists. Therefore, a new image acquisition would be realized by different operators that will not follow the same initial setup (especially, in terms of camera network and illumination conditions). Finally, in both image acquisitions a simple tape is used to measure few lengths that are adopted as references to metrically scale the photogrammetric model during the processing step.

\section{PROCESSING METHODOLOGY}

The methodology adopted for the 3D documentation of the crime scene and the rapid recording of its contamination is summarized in Figure 3 and further described in the following sections.

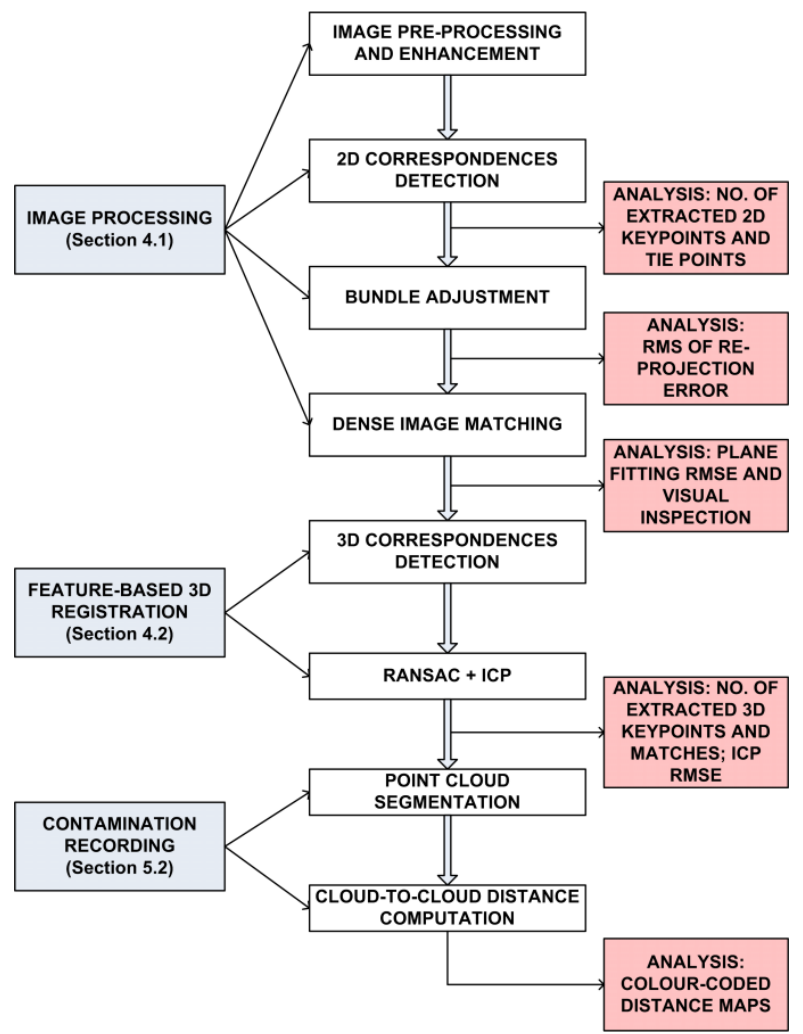

Figure 3. The workflow adopted in the project.

\subsection{Image processing}

The adopted image-based 3D reconstruction procedure follows the typical photogrammetric workflow, consisting of three main steps, namely: image correspondences detection, bundle adjustment and dense image matching. Although their level of automation is reaching high standards, an essential pre-requisite to support these tasks and improve the quality of the final results (especially in terms of precision, accuracy and reliability) is represented by the quality of the input images. This particularly applies when dealing with low-textured surfaces, repeated patterns, illumination changes and image problems like sensor noise and jpeg artefacts. A typical apartment's room like the "studio" simulated at the Crime Scene House, coupled with the use of a consumer-grade imaging sensor, is thus an example of a challenging scenario that can potentially benefit from the adoption of an ad-hoc 
image pre-processing step. Therefore, a pre-processing pipeline following the work presented in Gaiani et al. (2016) is here adopted to improve the quality of the acquired panoramas. After an initial histogram's stretching and white balancing, a de-noise processing using the $\mathrm{YCbCr}$ colour space, followed by an advanced image content enhancement technique, are applied. The latter adopts the well-known adaptive Wallis Filtering algorithm (Wallis, 1976), in its improved version implemented in the open-source photogrammetric tool GRAPHOS (González-Aguilera et al., 2016), available at https://github.com/itos3d/GRAPHOS. The filter parameters (namely, contrast, brightness, window size, mean and standard deviation) were accurately selected in accordance with the characteristics of the dataset. A qualitative comparison between an original and corresponding pre-processed panorama is shown in Figure 4.

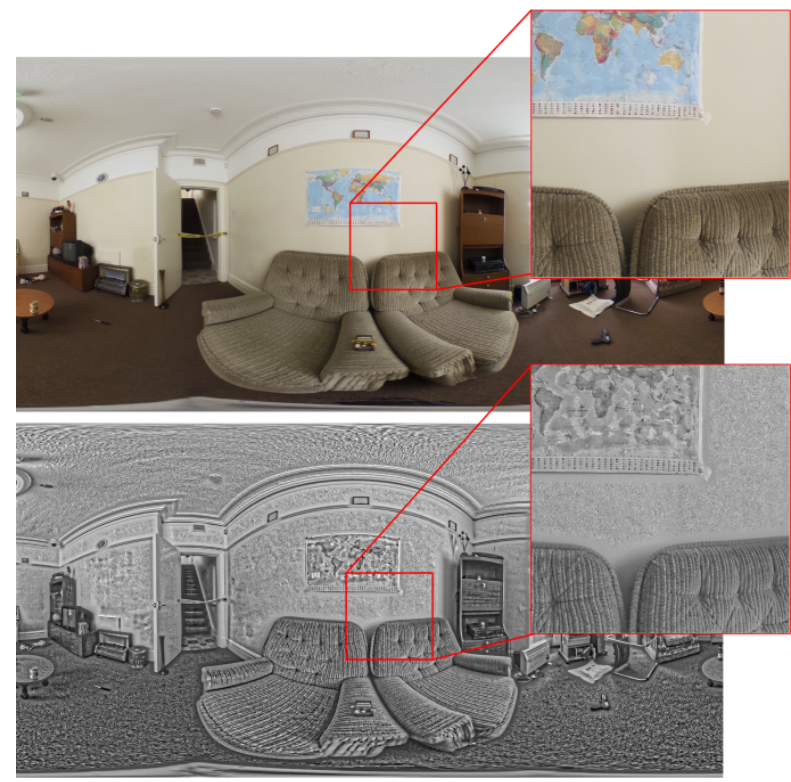

Figure 4. Example of the image pre-processing pipeline: original panorama (above) and pre-processed one (bottom).

Starting from the pre-processed panoramas, the photogrammetric pipeline is applied, employing a bundle adjustment with a spherical camera model. The RMS (Root Mean Square) of the re-projection errors is used to evaluate the image space error (precision of the image triangulation).

Results listed in Table 2 show the improvement gained by the application of the pre-processing step as support to the photogrammetric pipeline. Although the number of keypoints is lower, they are more likely to be correctly matched (i.e. the number of final tie points increases) and their distribution in image space is better balanced throughout the image (including points matched on floor, ceiling, walls and cabinets, see red dots in Figure 5, bottom). This leads to a higher precision in image space, as showed by the lower RMS of re-projection errors (third row of Table 2).

\begin{tabular}{|c|c|c|}
\cline { 2 - 3 } \multicolumn{1}{c|}{} & $\begin{array}{c}\text { w/o pre- } \\
\text { processing }\end{array}$ & $\begin{array}{c}\text { with pre- } \\
\text { processing }\end{array}$ \\
\hline No. of keypoints & 15,957 & 12,653 \\
\hline No. of tie points & 7,085 & 10,963 \\
\hline Img. space error (pixel) & 0.82 & 0.51 \\
\hline $\begin{array}{c}\text { No. of 3D points in the } \\
\text { dense point cloud }\end{array}$ & $\sim 12$ million & $\sim 9$ million \\
\hline
\end{tabular}

Table 2. Scenario A: results of image orientation without (left) and with (right) image pre-processing.
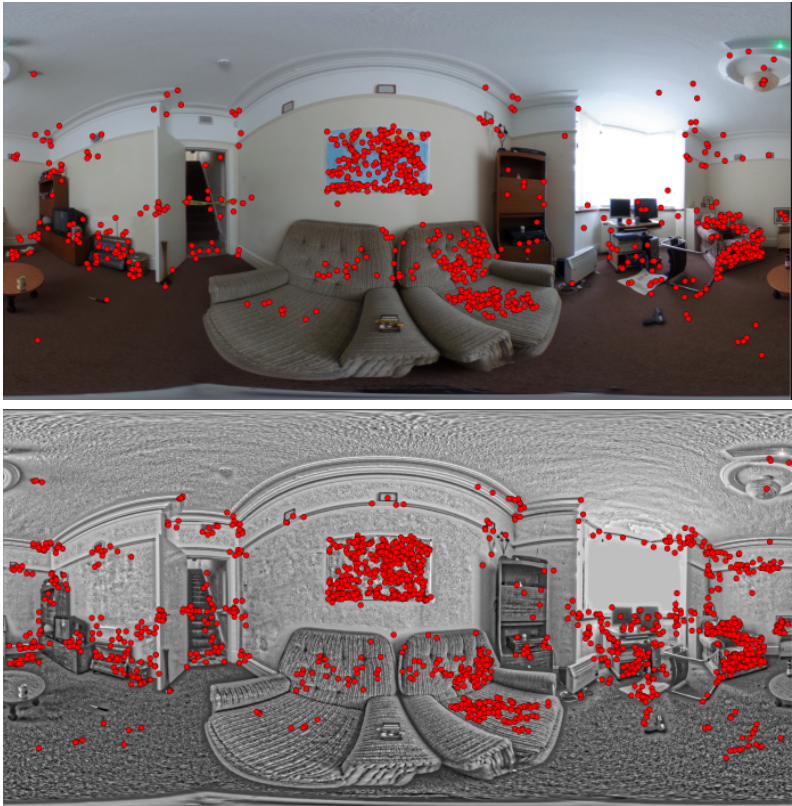

Figure 5. Example of tie point (red dots) distribution without (top) and with (bottom) image pre-processing.

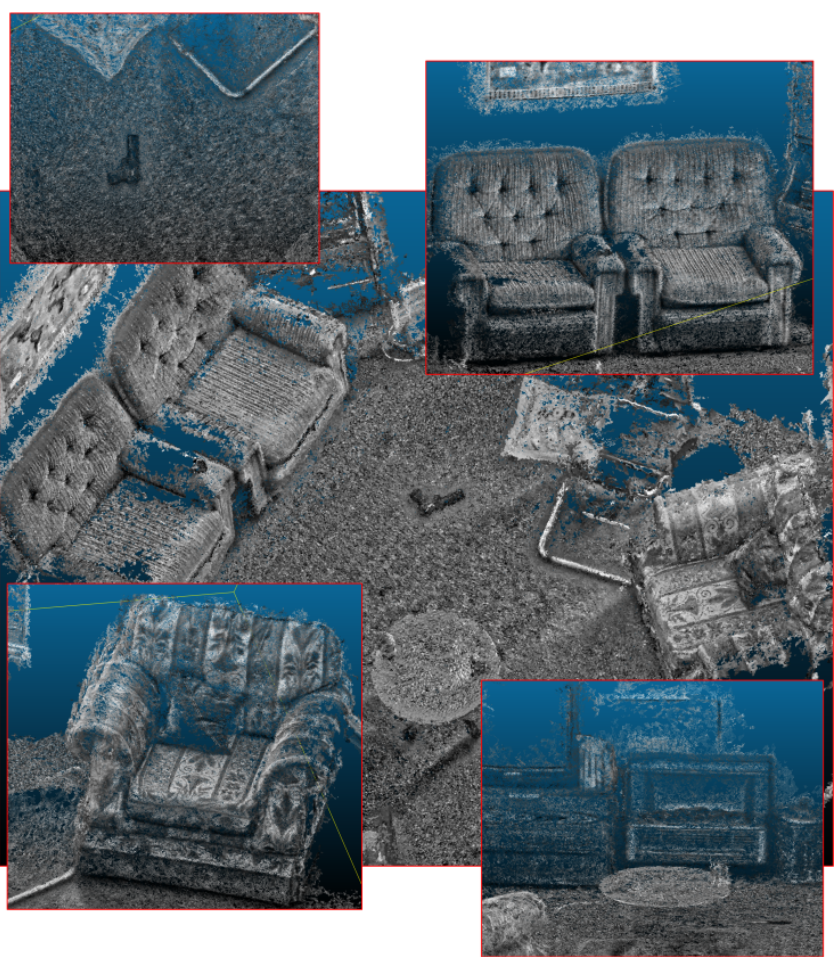

Figure 6. An overview and detailed views of the 3D dense point cloud (Scenario A).

Finally, starting from the estimated camera poses, a dense 3D reconstruction via pixel-based image matching algorithm is carried out. This is performed using the first-level image pyramid, corresponding to the original full image resolution. The derived dense point cloud features a mean spatial resolution in the range of 1 to $5 \mathrm{~mm}$ and consists of more than 9 million points (Figure 6). The number of matched 3D points increases when the original panoramas are used as input (see last row in Table 2), however this actually leads to more noisy results when points are matched on textureless surfaces (see Section 5.1). 


\subsection{Feature-based automatic 3D registration}

The automatic image-based 3D reconstruction described in Section 4.1 is then applied to both datasets (pre- and postmisplacement of evidence), leading to the generation of two point clouds corresponding to the pre-contamination (Scenario A) and post-contamination (Scenario B) crime scene scenarios. Although metrically consistent (i.e. scaled), the point clouds are referred to different local coordinate systems and, therefore, cannot be directly compared to each other. A feature-based automatic 3D registration is thus adopted for data alignment, based on a two-step procedure:

- coarse registration, using 3D feature correspondences, automatically detected on the input point clouds;

- fine registration, using an ICP-like refinement.

Although several methods have been recently investigated for automatic data registration and fusion using 3D invariant features (Salti et al., 2011; Tombari and Remondino, 2013; Hänsch et al., 2014), most of the adopted algorithms are still based on an interactive approach, i.e. they require manually selected correspondences for a point-to-point initial registration. In this study, although the amount of data is limited, a completely automatic procedure is adopted using as input the uppermost area of the point clouds, i.e. those portions (upper sections of walls and ceiling) where no reasonable suspect of contamination exists and thus more unlikely to be changed. This allows (i) to avoid any user manual interaction, that could lead to erroneous points identification given the limited number of easily recognizable details in the common areas, (ii) to support non-expert users with a fully automatic process, and (iii) to make the overall pair-wise registration process faster, in view also of a future extension of the method to higher number of point clouds (e.g., various contamination epochs).

The resulting methodology, summarized in Figure 7, is entirely performed in Cloud Alignment Workbench (CLAW), a tool within the GRAPHOS open-source photogrammetric suite and specifically developed for automatic pairwise registration:

- Detection: 3D keypoints are extracted out of each point cloud in order to detect a subset of distinctive and repeatable 3D points. Among the several 3D keypoint detectors proposed in the literature (Tombari et al., 2013) and available in CLAW, the Intrinsic Shape Signature (ISS) detector (Zhong, 2009) is used as various tests proved its good performance with the datasets under investigation.

- Description: once keypoints are detected, each extracted point is then associated with a compact representation of its neighbourhood by applying a 3D local descriptor. Many keypoint local descriptor have been developed (Aldoma et al., 2012) and, among the ones included in CLAW, the Fast Point Feature Histograms (FPFH, Rusu et al., 2009) is adopted. Indeed, it showed the best performance in terms of trade-off between accuracy and speed.

- Correspondences estimation: a pairwise matching of 3D features is performed using a nearest-neighbour search based on the Euclidean distance. This processed is supported by a correspondences rejection step (RANSAC approach; Fischler and Bolles, 1981), in order to eliminate false matches.

- Alignment: the outlier-free set of correspondences is used to estimate a 6 Degree-Of-Freedom (6-DOF) pairwise transformation (A-B), that is ultimately refined by ICP. The Root Mean Square Errors (RMSEs) after the ICP refinement step are listed in Table 3.

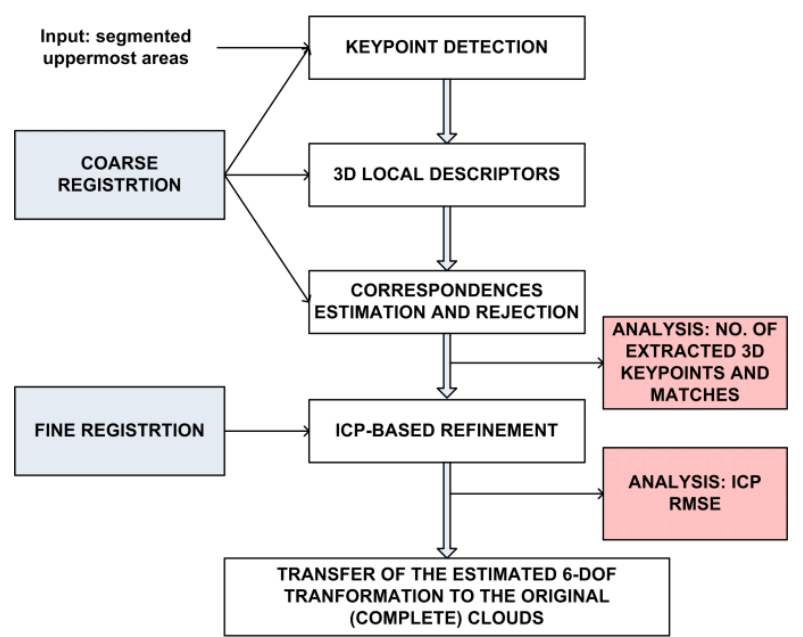

Figure 7. The adopted feature-based pipeline for pairwise point cloud registration.

The keypoints detected on the segmented point cloud of Scenario A are showed in Figure 8 (top, red points), whereas the $3 \mathrm{D}$ vectors connecting the correspondences (green points) matched between Scenario A and Scenario B, are drawn in grey in Figure 8 (bottom). Furthermore, the number of keypoints and correspondences (after the RANSAC-based rejection) detected for the pairwise registration is listed in Table 3.

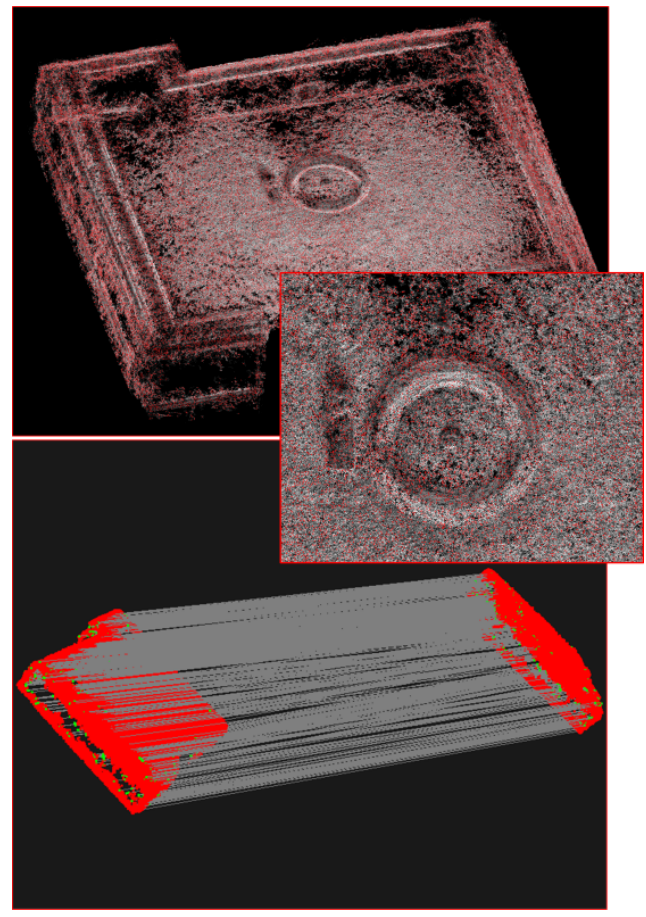

Figure 8. Keypoints extracted from Scenario A (top, red points) and outlier-free matches (green points) detected between

Scenario A and B. Grey rays represent the vectors linking the points matched between the two point clouds.

\begin{tabular}{|c|c|c|}
\cline { 2 - 3 } \multicolumn{1}{c|}{} & Scenario A & Scenario B \\
\hline No. keypoints/ & $105,172 /$ & $108,659 /$ \\
Tot. no. points & $3,866,300$ & $3,805,075$ \\
\hline No. matches ${ }^{*}{ }^{*}$ & \multicolumn{2}{|c|}{ A-B: 7,010} \\
\hline RMSE ICP $(\mathrm{mm})$ & \multicolumn{2}{|c|}{ A-B: 7.2} \\
\hline
\end{tabular}

Table 3. Results of automatic point clouds pairwise registration. ${ }^{(*)}$ after RANSAC 


\section{RESULTS}

\subsection{Evaluation of 3D reconstruction}

The quality of the final 3D reconstruction (i.e. 3D dense point clouds) is evaluated by (i) visually inspecting the amount of details that could be generated by the dense reconstruction step, and (ii) performing local comparisons with known reference shapes.

The former is aimed at assessing the performance of the tested low-cost system for the 3D geometric reconstruction of small objects, that may provide evidence for the investigation. Figure 9 shows three detailed views of the dense point clouds extracted from the original (left) and pre-processed (right) panoramas.

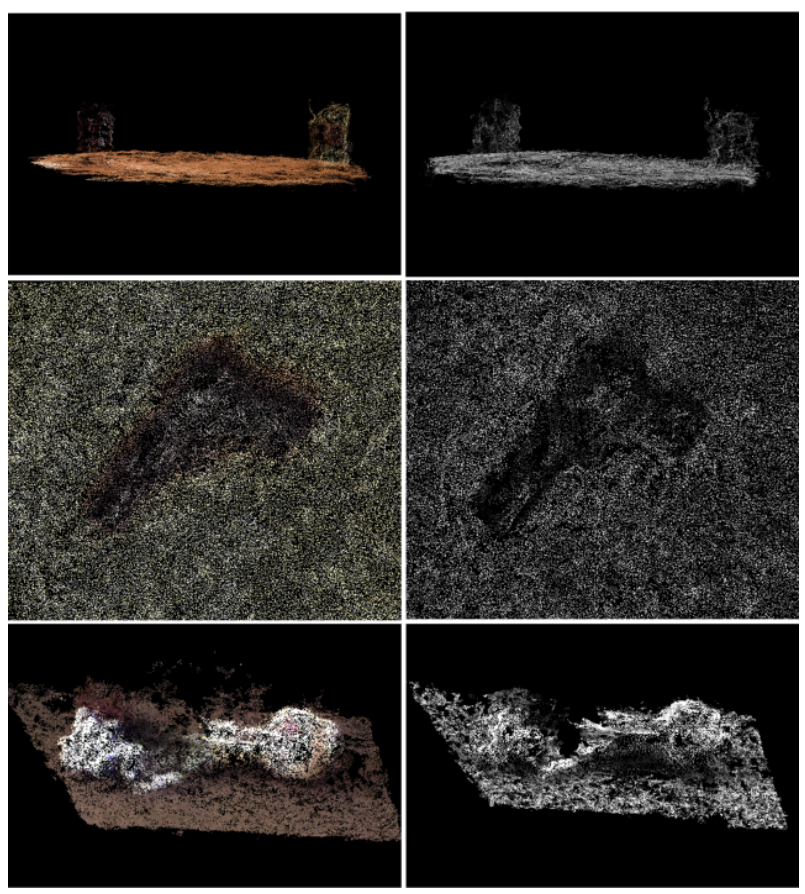

Figure 9. Examples of objects reconstructed in 3D with original (left) and pre-processed (right) panoramas.

Both approaches (with and without image pre-processing) are able to match points on small objects (like the gun, the bottle, the can and the vase), providing for a $3 \mathrm{D}$ documentation of the location and shapes of these artefacts. However, both 3D reconstruction procedures fail when dealing with smaller objects (e.g. the packet of cigarettes and the lighter, see Figure 10), that can hardly be distinguished in the point clouds.

In order to metrically evaluate the noise affecting the point clouds and measure the deviation of the matched points from a "reference" parametric shape, planar patches are segmented and compared against their corresponding best-fit planes. Figure 11 shows the planar patches extracted from both low-textured (walls, ceiling and cabinets) and textured surfaces (floor and map). The plane fitting RMSEs are finally listed in Table 4 . The effect of the panoramas' pre-processing is evident when lowtextured surfaces are analysed (green rows in Table 4). Although a lower number of points is matched when preprocessed images are used (as also showed from the total number of 3D points listed in Table 2 - last row), those points are more precisely triangulated and the plane fitting RMSEs are significantly lower, if compared to the ones estimated from the point clouds derived from original panoramas.

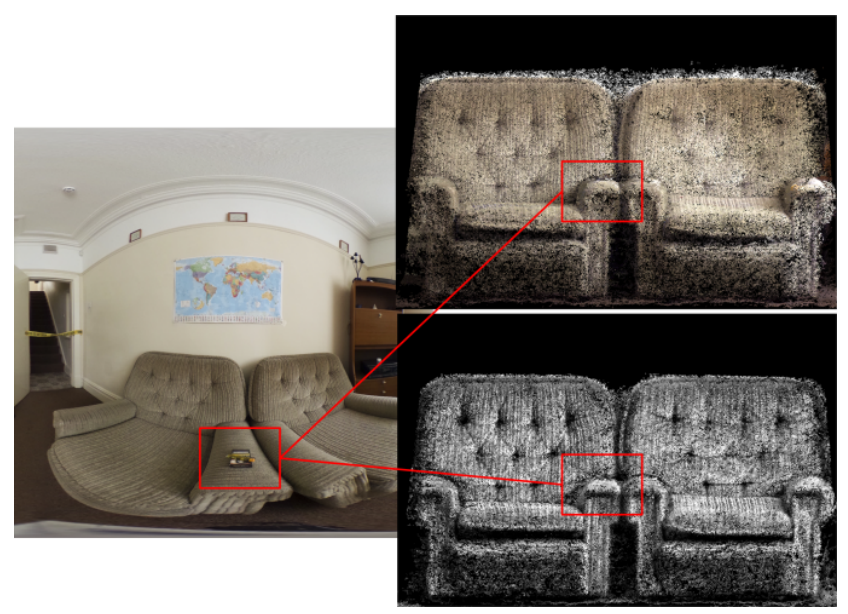

Figure 10. Example of objects which are not reconstructed in 3D neither with original (top) nor with pre-processed (bottom) panoramas.

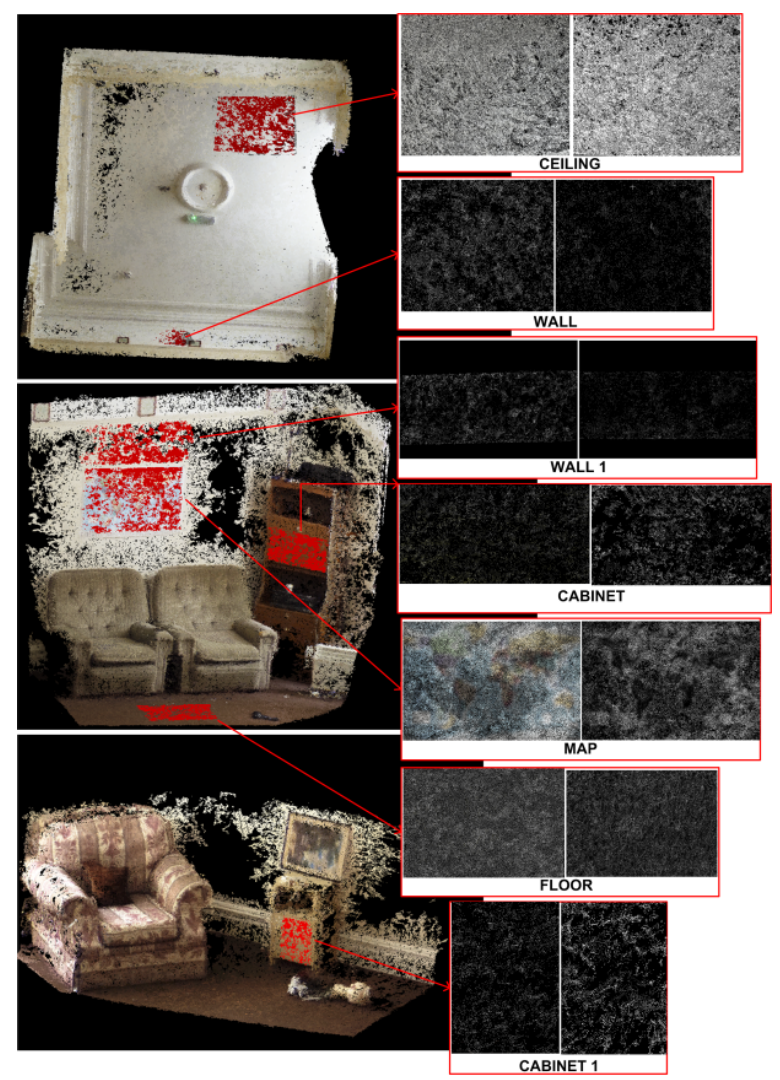

Figure 11. Patches segmented from the point clouds derived from original (left) and pre-processed (right) panoramas.

\begin{tabular}{|c|c|c|}
\cline { 2 - 3 } \multicolumn{1}{c|}{} & Original & Pre-processed \\
\hline Map & 3 & 3 \\
\hline Floor & 2 & 2 \\
\hline Ceiling & 14 & 7 \\
\hline Wall & 21 & 15 \\
\hline Wall 1 & 26 & 15 \\
\hline Cabinet & 23 & 9 \\
\hline Cabinet 1 & 27 & 16 \\
\hline
\end{tabular}

Table 4. Plane fitting RMSEs [mm]. 


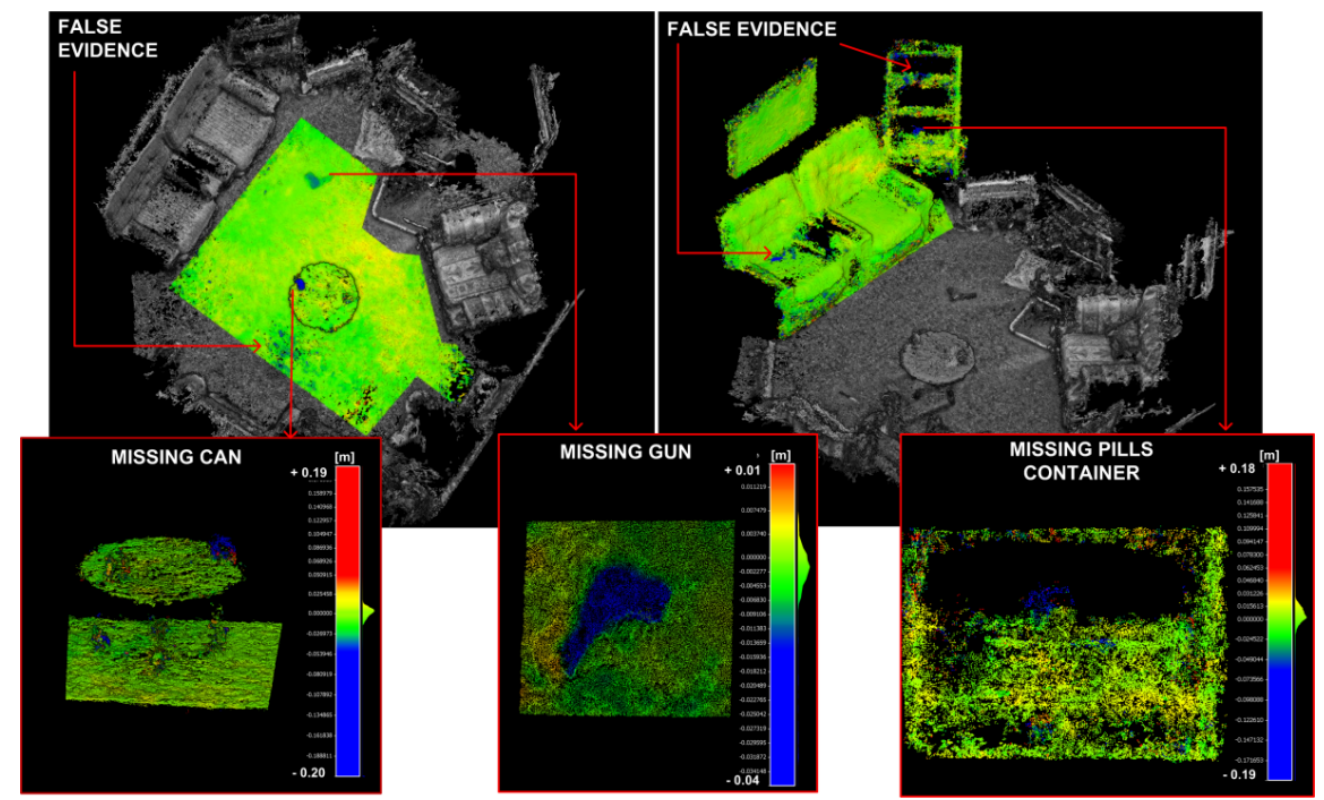

Figure 12. Colour-coded distance maps delivered by comparing pre- and post-contamination scenarios: results computed on large segmented areas (top) are then used to further analysed the suspected changes (bottom).

\subsection{Evaluation of contamination}

In order to identify suspected contamination, the automatically registered point clouds of Scenario A (pre-contamination) and Scenario B (post-contamination) are finally compared to each other. Given the amount of data compared to the small entity of the expected changes, a manually segmentation step is first carried out, by splitting the data into four main areas. Then, for each segmented area, cloud-to-cloud signed distances are automatically computed adopting the method Multiscale Model to Model Cloud Comparison (Lague et al., 2013). This algorithm, implemented in the open source software CloudCompare (http://www.danielgm.net/cc/), performs a direct comparison of point clouds in $3 \mathrm{D}$, thus avoiding the preliminary phase of meshing or gridding.

In our tests, each point of the Scenario A data is selected as "core" point and used to extract the distance between the two clouds. The colour-coded maps delivered by inter-comparison are then visually analysed to identify any clue of contamination. For instance, two examples of maps are showed in Figure 12 (top): while the majority of points is associated with null distances (green colour), few blues spots (corresponding to "negative" distances, i.e. missing features) are evident. Those spots are then segmented out of the original cloud and finally deepen analysed by stretching the histogram of their colourcoded distances. This leads to two possible cases:

- "true" missing objects, like the can, the gun and the pills container (Figure 12, bottom) that are reconstructed in Scenario A and missing in Scenario B (i.e. evidence of crime scene contamination);

- "false" evidence, i.e. positive or negative distances due to noise affecting the point clouds and thus not corresponding to any actual change occurred between the two scenarios (see the noise on the floor, on the armchair and on the cabinet, all marked as "false evidence" in Figure 12).

\section{CONCLUSIONS}

This work investigated the use of a low-cost imaging sensor, the Ricoh Theta spherical camera, combined with the adoption of algorithms for image pre-processing, photogrammetric 3D reconstruction, data registration and analysis, all implemented in open-source or low-cost software solutions. The contribution of the paper is twofold. First, it evaluated the performance of the tested system for the rapid 3D reconstruction of a forensic scenario, i.e. a crime scene simulated in a typical apartment's room. Both qualitative analysis (i.e. visual inspection of reconstructed objects) and quantitative evaluation (i.e. bestfitting plane analysis on various surfaces) showed the good potential of the method for the $3 \mathrm{D}$ documentation of the crime scene. A dense 3D point cloud (mean spatial resolution in the range of 1 to $5 \mathrm{~mm}$ ) locally characterized by limited plane fitting RMSE (always below $2 \mathrm{~cm}$, when the pre-processing is performed) is indeed achieved. Furthermore, small objects like a gun, a bottle, a can, a vase and a pills container, could be reconstructed and, thus, potentially documented as investigation evidence. However, the approach fails when it comes to the modelling of smaller objects, like a packet of cigarettes, a lighter, bullet and a knife: in these cases and, more generally, when a higher level of detail and accuracy is needed, more advanced image-based and range-based systems are still required, to lead to a high-quality $3 \mathrm{D}$ reconstruction. Currently, there is no recognized worldwide limit for the accuracy required in crime scene investigation. While in the UK there is no published standard, in the USA an accepted accuracy limit of 0.25 inch is applied (Lothridge, 2013).

Second, the paper proposed a methodology for the rapid recording of possible contaminations which could have changed the original appearance of the crime scene. The approach, based on an automatic 3D feature-based point cloud registration and cloud-to-cloud distance computation, was able to successfully identify the biggest objects among the intentionally removed ones. Furthermore, it can be easily extended to different types of contaminations, e.g. the displacement of objects from their original position within the crime scene. However, two limitations are evident and should be dealt with in the future. The approach is not completely automatic and a manual segmentation of the point cloud is needed for the distance computation step to be effective. Finally, the noise of the data can negatively affect the analysis, leading to the detection of false evidence. The approach will be thus improved in the next future, by supporting the analysis of the reconstructed 3D geometry with (RBG) information available on the original panoramas. 


\section{ACKNOWLEDGEMENTS}

The project has been developed in the framework of the EU "Marie Sklodowska-Curie Action" Digital Forensic Archaeology (Dig-For-Arch), Grant Agreement No. 708974.

\section{REFERENCES}

Aghayaria, S., Saadatsereshta, M., Omidalizarandi, M., Neumannb, I., 2017. Geometric Calibration of Full Spherical Panormamic Ricoh-Theta Camera. In: ISPRS Annals of the Photogrammetry, Remote Sensing and Spatial Information Sciences, Vol. 4(1/W1), pp. 237-245.

Aldoma, A., Marton, Z.C., Tombari, F., Wohlkinger, W., Potthast, C., Zeisl, B., Rusu, R.B., Gedikli, S., Vincze, M., 2012. Point Cloud Library: Three-dimensional object recognition and 6 DOF pose estimation. In: IEEE Robotics and Automation Magazine (RAM), Vol. 19(3), pp. 80-91.

Amiri Parian, J., Gruen, A., 2010. Sensor modeling, selfcalibration and accuracy testing of panoramic cameras and laser scanners. In: ISPRS Journal, Vol. 65(1), pp. 60-76.

Barazzetti, L., Previtali, M., Roncoroni, F., 2017. 3D Modelling with the Samsung Gear 360. In: The Int. Archives of Photogrammetry, Remote Sensing and Spatial Information Sciences, Vol. 42(2/W3), pp. 85-90.

Buck, U., Naether, S., Rass, B., Jackowski, C., Thali, M.J., 2013. Accident or Homicide - Virtual crime scene reconstruction using 3D methods. In: Forensic Science International, Vol. 225(1-3), pp. 75-84.

Dustin, D., Liscio, E., 2016. Accuracy and repeatability of the Laser Scanner and Total Station for crime and accident scene documentation. In: J. Assoc. Crime Scene. reconstr. 2016; pp.57-67.

Dutelle, A.W., 2016. An Introduction to Crime Scene Investigation. Jones \& Bartlett Publishers ( $3^{\text {rd }}$ Edition), pp. 645.

Fangi, G., 2007. The multi-image spherical panoramas as a tool for architectural survey. In: The Int. Archives of Photogrammetry, Remote Sensing and Spatial Information Sciences, Vol. 36(5/C53), pp. 311-316.

Fangi, G., 2009. Further developments of the spherical photogrammetry for cultural heritage. In: XXII International Committee for Cultural Heritage (CIPA), pp. 11-15.

Fischler, M.A., Bolles, R.C., 1981. Random Sample Consensus: a paradigm for model fitting with applications to image analysis and automated cartography. In: Communications of the ACM, Vol. 24(6), pp. 381-395.

Gaiani, M., Remondino, F., Apollonio, F.I. and Ballabeni, A., 2016. An advanced pre-processing pipeline to improve automated photogrammetric reconstructions of architectural scenes. In: Remote Sensing, Vol. 8(3), 178.

González-Aguilera D., López, L., Rodriguez-Gonzalvez, P., Guerrero, D., Hernandez-Lopez, D., Remondino, F., Menna, F., Nocerino, E., Toschi, I., Ballabeni, A., Gaiani, M., 2016. Development of an all-purpose free photogrammetric tool. In: The Int. Archives of Photogrammetry, Remote Sensing and Spatial Information Sciences, Vol. 41(B6), pp.31-38.

Hänsch, R., Weber, T. and Hellwich, O., 2014. Comparison of 3D interest point detectors and descriptors for point cloud fusion. In: ISPRS Annals of the Photogrammetry, Remote Sensing and Spatial Information Sciences, Vol. 2(3), pp.57-64.

Holowko, E., Januszkiewicz, K., Bolewicki, P., Stinik, R., Michonski, J., 2016. Application of multi-resolution 3D techniques in crime scene documentation with bloodstain pattern analysis. In: Forensic Science International, Vol. 267, pp. 218-227.
Jackson, A.R.W. and Jackson, J., 2008. Forensic Science. Pearson Education (2 ${ }^{\text {nd }}$ Edition), pp. 468.

Lague, D., Brodu, N., Leroux, J., 2013. Accurate 3D comparison of complex topography with terrestrial laser scanner: Application to the Rangitikei canyon (N-Z). In: ISPRS Journal of Photogrammetry and Remote Sensing, Vol. 82, pp. 10-26.

Liscio, E., Hayden, A., Moody, J., 2016. A comparison of the terrestrial Laser Scanner and Total Station for Scene Documentation. In: J. Ass. Crime Scene. reconstr. 2016, pp.1-8. Lothridge, K., 2013. A Guide for Law Enforcement, https://www.nist.gov/sites/default/files/documents/forensics/Cri me-Scene-Investigation.pdf (last accessed on 20/10/2017).

Matzen, K., Cohen, M. F., Evans, B., Kopf, J., Szeliski, R., 2017. Low-Cost 360 Stereo Photography and Video Capture. In: Journal ACM Transactions on Graphics, Vol.36(4), pp. 148. Perfetti, L., Polari, C., Fassi, F., 2017. Fisheye photogrammetry: tests and methodologies for the survey of narrow spaces. In: The Int. Archives of Photogrammetry, Remote Sensing and Spatial Information Sciences, Vol. 42(2-W3), pp. 573- 580.

Rusu, R.B., Blodow, N. and Beetz, M., 2009, May. Fast point feature histograms (FPFH) for 3D registration. In: Robotics and Automation, 2009. ICRA'09. IEEE International Conference on, pp. 3212-3217.

Salti, S., Tombari, F. and Di Stefano, L., 2011. A Performance Evaluation of $3 \mathrm{D}$ Keypoint Detectors. In: $3 D$ Imaging, Modeling, Processing, Visualization and Transmission (3DIMPVT), pp. 236-243.

Sakharkar, V.S., Gupta, S.R., 2013. Image Stitching Techniques-An overview. In: International Journal of Computer Science and Applications, Vol. 6(2), pp. 324-330.

Schneider D., Maas H.-G., 2003. Geometric Modelling and Calibration of High Resolution Panoramic Camera, In: Optical 3-D Measurement Techniques, Vol. II, pp. 122-129.

Shepppard, K., Cassella, J.P., Fieldhouse, S., 2017. A comparative study of photogrammetric methods using panoramic photography in a forensic contest. In: Forensic Science International, Vol. 273, pp. 29-38

Szeliski, R. and Shum, H., 1997. Creating full view panoramic image mosaics and environment maps. In: Proceedings of SIGGRAPH, pp. 251-258.

Thali, M.J., Braun, M., Bruschweiler, W., Dirnhofer, R., 2000. Matching tire tracks on the head using forensic photogrammetry. In: Forensic Science International, Vol. 113(1-3), pp. 281-287

Tombari, F. and Remondino, F., 2013. Feature-based automatic 3D registration for cultural heritage applications. In: Digital Heritage International Congress, Vol. 1, pp. 55-62.

Tombari, F., Salti, S., Di Stefano, L., 2013. Performance evaluation of 3D keypoint detectors. In: Int. Journal of Computer Vision, Vol. 102(1-3), pp. 198-220.

Wallis, R., 1976. An approach to the space variant restoration and enhancement of images. In: Proc. of the Symposium on Current Mathematical Problems in Image Science, pp. 329340 .

Zancajo-Blazquez, S., Gonzalez-Aguilera, D., HernandezLopez, D., 2015. An automatic image-based modelling method applied to Forensic Infography. https://doi.org/10.1371/journal.pone.0118719 (last accessed on 20/10/2017).

Zhong, Y., 2009. Intrinsic shape signatures: A shape descriptor for 3D object recognition. In: Proc. ICCV, pp. 1-8. 